\title{
HACIA UNA EXTENSIÓN DE CALIDAD: LOS SUPUESTOS DE UNA EXPERIENCIA
}

Silvia Pellegrini*

* Vicerrectora de Comunicaciones y Extensión de la Pontificia Universidad Católica de Chile 


\section{RESUMEN}

La extensión aparece como el desafío de la universidad del futuro. El cambio cultural y social representado por el proceso globalizador ha afectado a las universidades en tanto la sociedad reclama de ellas generar una forma eficiente de acompañamiento cultural.

$\mathrm{Su}$ calidad no se mide, entonces, por su contenido específico ni por la capacidad de adicionar recursos a la actividad académica, sino por lograr una adecuación precisa que redunde en desarrollo, tanto de la sociedad como de la institución universitaria. No es posible separar una extensión de calidad de una concepción de la función social del saber y del rol que en ésta cabe a la universidad.

La experiencia de la Pontificia Universidad Católica de Chile refleja la variedad y amplitud en las que puede desarrollarse esa relación.

\section{ABSTRACT}

Outreach activities appear as the challenge for the university of the future. The cultural and social change involved in globalization has affected universities while society demands that they prepare an efficient form of cultural supplementation.

Thus, university quality is not measured by their specific contents or by their ability to add resources to academic activities, but by their ability to achieve a precise adaptation resulting in the development of both society and universities as institutions. It is not possible to separate the extension of quality from a concept of the social function of knowledge and the role that universities are called upon to play in this respect.

The experience of the Pontificia Universidad Católica de Chile reflects the variety and extent to which this relationship can take place. 


\section{HACIA UNA EXTENSIÓN DE CALIDAD: LOS SUPUESTOS DE UNA EXPERIENCIA}

Hacia una extensión de calidad

Hablar de extensión de calidad requiere, en primer lugar, precisar el concepto de extensión, por cuanto éste es el menos definido y delimitado de la trilogía clásica de la actividad universitaria: docencia, investigación y extensión. La primera ostenta el valor de lo tradicional: no hay universidad sin alumnos, sin transmisión del conocimiento en el aula. Para muchos, este es el aspecto esencial de la institución de educación superior, y esa es la razón por la cual muchas universidades se definen sólo como instituciones docentes. La investigación, por su parte, toma el valor de la renovación, de la generación del conocimiento; de allí surgen planteamientos tales como "no hay docencia sin investigación" o "la investigación es el alma del trabajo universitario". Pasa a ser, entonces, el elemento sustentador de la docencia, aquello que le da validez a su contenido. Y lograr la proporción adecuada entre esas actividades se hace un tema constante de la gestión universitaria, tanto como las rivalidades entre los defensores de una u otra como la esencial y prioritaria.

Éstos son los dos pilares fundamentales en la mayoría de los procesos de evaluación de académicos y en la propia estimación de su trabajo ${ }^{1}$. La extensión, en cambio, se mira como actividad menor. Ningún académico se justifica en ella. Más aún, generalmente se la percibe como distante de los "verdaderos intereses universitarios" y muchos profesores la ven en confrontación -al menos temporal- con las restantes actividades que parecen más relacionadas con su vocación central hacia el saber.

1 Por lo demás, la demanda más habitual de los profesores radica en tener más tiempo para investigar. 
Sin duda la mayor integración de la extensión se da en el área artística, donde se la considera como parte ineludible de la labor académica. Desde los otros sectores universitarios se la percibe plenamente justificada sólo en el arte y -a lo más- se la asocia a la posibilidad de incrementar los recursos materiales a través de la venta de servicios a terceros, un modo también distante de la cultura académica generalizada.

Hoy, sin embargo, la extensión aparece como el desafío de la universidad del futuro. La situación de cambio cultural y social representado por el proceso globalizador ha afectado a las universidades de un modo diverso a lo que hicieron sus anteriores procesos de desarrollo: esta vez, las demandas no provienen de grupos internos, sino de la sociedad que reclama la posibilidad de participar de su acción y de su saber. Este requerimiento ha sido impulsado, en parte, por la rapidez con que se genera el nuevo conocimiento y por la consiguiente necesidad -siempre en aumento- de educación continua y reciclaje profesional. Influye también la expectativa de lograr mejores condiciones de vida a través de la actualización de los saberes. La universidad recibe, en ese contexto, la demanda de establecer una relación permanente con la sociedad, de generar una forma eficiente de acompañamiento cultural para el proceso de cambio.

La nueva perspectiva social tiene plena validez, tanto por la labor cultural propia de las instituciones universitarias como por el rol especial de credibilidad que les asigna el país. Pero no cabe duda que se ven enfrentadas a un escenario diferente que requiere de reflexión y adecuación en sus tareas futuras y, por ende, en sus planes de desarrollo. Y como la perspectiva no es volver la mirada hacia sí mismas, sino al exterior, se trata, en síntesis, de establecer una política de extensión.

Las relaciones universidad - sociedad

El desafío es significativo. Se trata de definir la extensión como la relación entre universidad y sociedad. Y su calidad, por ende, está determinada por lo adecuada y fructífera que esa relación sea para ambas. Extensión se entiende así no cómo el agregado ilegítimo de una actividad principal, sino como la ampliación del rango de la acción 
universitaria desde un ámbito reservado y con un público cautivo (el estudiante) hacia un espacio de márgenes imprecisos, con un grupo humano más variado y fluctuante, y del cual es necesario extraer elementos enriquecedores para la propia acción. Esta alternativa implica un significativo cambio de concepto: hacer extensión significa ahora mantener los objetivos propios de la actividad universitaria, (generar, conservar y transmitir conocimiento) en una relación con un conjunto diferente de personas, que tiene con la institución vínculos menos formales y estables en el tiempo, y al que hay que atraer con una oferta que perciba adecuadamente lo que es de utilidad para su propio desarrollo.

Una extensión de calidad no se mide, entonces, por las bondades de su contenido específico, como sería la forma tradicional (un buen concierto, una excelente exposición de arte, una gran conferencia), tampoco por la capacidad o habilidad para adicionar recursos a la actividad académica principal, sino por lograr una adecuación precisa de los márgenes de acción que redunde en desarrollo tanto de la sociedad como de la institución universitaria. No es posible separar una extensión de calidad de una concepción de la función social del saber y del rol que en ésta cabe a la universidad ${ }^{2}$.

Las experiencias históricas demuestran que el vínculo universidad-sociedad ha sido bastante tenso, en tanto ha fluctuado entre, por una parte, percibir a la universidad como "la torre de cristal", caracterizada por el aislamiento, y, por otra, subordinarse al imperio de las ideologías, cuando se impone la lógica de la polis en la búsqueda y aplicación del conocimiento. Los riesgos de esta segunda alternativa son los que han hecho deseable para muchos la primera; pero, en el actual contexto, donde el conocimiento está inevitablemente expuesto y accesible, lograr una relación equilibrada entre ambas parece no sólo necesario, sino imprescindible para la sobrevivencia de la institución universitaria ${ }^{3}$.

2 "Toda política de extensión implica una concepción de la función social del saber y corresponde a la idea que se tenga de las relaciones que deben existir entre la Universidad y la Sociedad." Ricardo Krebs W. "Historia de las Políticas de Extensión de la PUC.", p. 207.

3 "Una universidad aislada intelectualmente de su contexto social, centrada en sí misma, está condenada a morir de inanición espiritual o a ser vencida por las circunstancias." Dr. Pedro Pablo Rosso, rector de la Pontificia Universidad Católica de Chile. Cuenta anual 2002. 
Podrían entonces sintetizarse los principios básicos de una extensión de calidad en los siguientes objetivos:

1. La proyección en la cultura y la significación de los temas: difundir en la sociedad los principios que inspiran a la universidad y a todas sus áreas de interés, a través de aportar conocimientos a la reflexión y significación de los temas sociales, como un modo de contribuir a la formación de la identidad y cultura del país ${ }^{4}$. Esta meta requiere una mirada alerta sobre los problemas culturales y la búsqueda de modos de acercamiento compatibles con una acción esencialmente académica. Es en este plano donde se inserta también la difusión artística, complementada con los otros ámbitos del saber.

2. El vínculo con otros actores sociales: establecer relaciones entre el conocimiento universitario y las necesidades de diversos actores de la sociedad, así como extraer de ella inspiración para generar nuevas líneas de docencia e investigación.

3. Las variadas formas de acercamiento temático, propiciando la mirada pluridisciplinaria sobre problemas complejos. En tanto la extensión tiene la condicionante de relacionarse de modo efectivo al diagnóstico y la acción frente a problemas complejos, requiere habitualmente la participación de diversas disciplinas. Así, puede contribuir al conocimiento, aprecio mutuo y trabajo conjunto entre ellas.

4. La transferencia de conocimientos a grupos extensos: generar docencia para que los nuevos conocimientos y el desarrollo de competencias se encuentre disponible para amplios grupos de la sociedad. El propósito es ofrecer acompañamiento para la formación constante durante la vida adulta y una actualización permanente a los profesionales. La línea futura de acción, en este ámbito, tiene que ver con el uso de nuevas metodologías que combinen modos presenciales y a distancia

5. La correspondencia de intereses y calidades con las otras actividades de la academia, requiere que la extensión sea la dimensión pública de aquello que las facultades realizan como proyecto de docencia e investigación, de modo de mantener los niveles de calidad y la interrelación entre los ámbitos de la trilogía universitaria. La

4 "La PUC, por la labor que le es propia, contribuye a la consolidación de modos de pensar y de hacer, susceptibles de ser entregados a la comunidad como elementos formativos de la identidad y cultura de todo el país." Consejo Superior. Políticas de Comunicación y Extensión 2000. 
extensión es, a su vez, un vehículo útil y efectivo para la incorporación de temáticas nuevas a la docencia e investigación, y el fomento de la asociación pluridisciplinaria. Una extensión de calidad no es un proyecto de gestión central, sino que requiere estar entroncado en las comunidades propias de cada saber y ser parte constitutiva de su concepción y desarrollo.

\section{La experiencia de la Pontificia Universidad Católica}

Hay pocos ejemplos de una institución de educación superior tan vinculada a su sociedad como la Pontificia Universidad Católica de Chile. A través de una gran variedad de formas e iniciativas ha llegado a tener una complejidad inmensa, a la que se suma una cantidad siempre creciente de proyectos. La responsabilidad última del contenido de la extensión radica en las facultades, y corresponde especialmente a la Dirección Superior la detección de necesidades, la estimulación de iniciativas y el vínculo entre facultades y disciplinas. Por otra parte, la extensión depende en una proporción muy significativa de su autofinanciamiento, dado que las partidas principales del presupuesto central costean con dificultad los requerimientos de la docencia y la investigación.

No parece posible, en la brevedad de estas páginas, hacer un recuento de todo lo que se realiza; por esa razón se recurrirá a algunos ejemplos que sirvan de ilustración. Muchos de éstos están claramente vinculados con iniciativas académicas de docencia o investigación regulares. Otros, en cambio, corresponden a iniciativas que se han generado al amparo de la Dirección Superior (ver Cuadro 1).

\section{Cuadro 1}

Actividades de la Vicerrectoría de Comunicaciones y Extensión 2003:

- 21 programas permanentes con 174 proyectos diferentes.

- Ingresos descentralizados de $\$ 4.423$ millones, destinados completamente a la realización de proyectos.

- Proyectos de extensión con participación de todas las unidades académicas de la universidad y 65 organismos externos.

- 608 eventos realizados en el Centro de Extensión para 160 entidades diferentes, con una asistencia de 317.444 personas.

- Publicación de 43 libros, 21 reediciones, 9 coediciones y 2 revistas trimestrales.

- 30.639 alumnos del Programa de Educación Continua. 


\section{La proyección en la cultura y la significación de los temas}

Desde siempre la universidad ha realizado una contribución significativa a la identidad nacional, inspirada en los valores que la sustentan. Hoy el principal desafío en este aspecto radica, precisamente, en proporcionar a la sociedad chilena elementos que le sirvan de interpretación y guía respecto de las otras sociedades con las que ha entrado abruptamente en contacto ${ }^{5}$. Este objetivo incluye el sostenimiento y promoción de los elementos significativos de la cultura nacional, tanto los de carácter autóctono ${ }^{6}$ como las tradiciones culturales propias del país y del resto de América Latina, como la difusión de conocimientos sobre aspectos de las culturas extranjeras, en especial de las vinculadas al país ${ }^{7}$.

Supone también un esfuerzo por reconocer aquellos rasgos, perspectivas o conocimientos que, siendo propios, puedan ser de interés y significación para otras culturas, lo que representa una posibilidad de trabajos conjuntos y asociaciones de diversa índole.

A modo de ejemplo, para capacitar a grupos indígenas dentro de los ámbitos de su propia cultura, la PUC creó Afunalhue: un centro de

5 En este aspecto se han ofrecido, durante el año recién pasado, diversos cursos y seminarios cuyos nombres revelan sus objetivos. Entre ellos cabe señalar: Capacitación y desarrollo para la empresa; Taller sobre divorcio; Taller de política agraria; Los vínculos familiares y su impacto en la sociedad; Ética, gestión pública y corrupción; Efectos civiles del matrimonio religioso; Chile, ¿un país para todos?; Comunidades de aprendizaje: Una nueva forma de aprender; Niños y hogares: Una realidad en conflicto; Taller de creatividad e innovación; Policía y comunidad: Los nuevos desafíos para Chile; Vivienda y ciudad; Vivienda saludable, por ejemplo. También ha generado exposiciones como: ¿Qué hace que los hogares de hoy sean tan diferentes, tan atractivos?; Chile: Un viaje en blanco y negro.

6 Las culturas autóctonas han sido una preocupación principal en el trabajo de la Pontificia Universidad Católica. Dispone de un Centro de Investigación para el Desarrollo de Pueblos Indígenas y de la sede regional Villarrica, donde se prepara a docentes para la etnia mapuche en un contexto sociocultural apropiado, que cuenta, incluso, con alojamiento y alimentación para los alumnos, en su mayoría de escasos recursos.

7 Genera permanentemente cursos de extensión destinados a estos aspectos, tales como "Perspectivas filosóficas de la antigüedad griega"; "Arte contemporáneo"; "El discurso: punto de encuentro de las humanidades". Monta exposiciones como: "Códices, mapas y manuscritos antiguos de España"; "Pintores rusos no oficiales"; "Fotos de China"; "Renania Palatinado"; "Ilustración británica"; "10 años de la ascensión al Everest"; "Amundsen y la conquista del Polo"; "Expresiones del Japón". Edita libros al estilo de "Lecturas anglosajonas"; "Castilla tajeada de sed como mi lengua"; "El traje: transformaciones de una segunda piel"; "Heidegger y Zubiri". Finalmente, difunde anualmente unos 30 ciclos de cine-arte donde se proyectan unas 200 películas de cine asiático, europeo, norte y latinoamericano. 
seminarios y reuniones construido al estilo de una ruca tradicional. En Afunalhue se cuenta también con una quesería, cuyos encargados -todos de la etnia mapuche- se han capacitado en Holanda y España. El proyecto cuenta con recursos provenientes del país vasco.

Un segundo ejemplo es el Programa de Artesanía Tradicional -dependiente de la Facultad de Arquitectura, Diseño y Estudios Urbanos- que mantiene un vínculo constante con los principales artesanos tradicionales del continente, a los que reúne anualmente en una Feria de Artesanía Tradicional. A lo largo de sus 30 años de existencia, la universidad ha ido formando una importante colección para la que se tienen diversas alternativas de exposición pública ${ }^{8}$.

En el convencimiento de que, para un desarrollo sustentable, la sociedad no puede prescindir de la colaboración de las universidades, ni éstas aislarse recurriendo solamente a sus propios intereses y capacidades, los proyectos de extensión tienen también como propósito generar un espacio de diálogo para el intercambio respetuoso que impulse a la acción. La iniciativa más reciente en este plano se denomina: "La UC mira a Chile"9.

En este mismo contexto se enmarca el propósito de la Revista Universitaria, que en los últimos años cambió su línea editorial desde perspectivas generales hacia el tratamiento de temas específicos de interés nacional, desde una mirada de especialidad universitaria.

8 Dentro de este propósito, se organizaron algunos congresos y simposios entre los que destacan las VI Jornadas Migratorias, el Congreso sobre Diversidad Cultural y la Temporada de la Cultura Tradicional, con actividades tales como teatro, música y cine.

Esta línea de trabajo ha realizado, asimismo, algunos cursos que plantean perspectivas humanas y culturales propias. Entre ellas: "Sanadores y chamanes"; "Lenguaje de los mitos"; "Rol político y social del arte en América Latina"; "Obras de arte contemporáneo"; "Psicología e identidad latinoamericana". Se ha reforzado también con algunas exposiciones, tales como "Telares" (una muestra de tejidos realizados de ese modo que reflejan las perspectivas, coloridos y formas de tantas culturas latinoamericanas), o "Rapa-Nui factor humano", la única exposición sobre Isla de Pascua realizada este año en el continente.

9 "La UC mira a Chile" es un programa de diálogos públicos entre profesores universitarios que incluye cuatro líneas diferentes: "Grandes Temas", para analizar, desde perspectivas diferentes, temas culturales genéricos, tales como el dolor, el cuerpo, la felicidad y la vida; "Actualidad", que entrega la mirada experta sobre temas puntuales, tales como las relaciones con los países vecinos, la crisis energética y el desarrollo de la minería; "Desde la Academia", para exponer las líneas de trabajo de diversos profesores que pudieran tener interés para público general o especializado, y "Desde el Aula", que da a conocer los resultados de las mejores tesis de alumnos que tengan esas mismas características. 
Algunos de sus dossier han tratado acerca del transporte en Santiago y los problemas de la educación y de la corrupción, por ejemplo ${ }^{10}$.

\section{El vínculo con otros actores sociales}

La extensión concebida como la relación universidad-sociedad requiere que la universidad reconozca los intereses propios de los diversos actores sociales y los considere en sus perspectivas.

En este aspecto, sirve de ejemplo el programa de Educación a Distancia TELEDUC que ha generado un modelo de trabajo con los más diversos organismos del país para la capacitación en espacios de Internet con acceso reservado a usuarios específicos. Esta metodología permite, incluso, cuantificar el acceso individual de cada uno de los participantes en los cursos $^{11}$.

Una iniciativa reciente, de enorme significación, ha sido el programa universidad-empresa, a través del cual la universidad, en estrecho contacto con diferentes grupos de empresas, ha generado diversas instancias de trabajo conjunto, de modo de adicionar recursos materiales y humanos para mejorar la calidad de los productos. Se ha creado una fundación que apoya el desarrollo de productos naturales, se han establecido alianzas estratégicas con la asociación de viñas, con la corporación de la madera y con la industria metal-mecánica, cuyos objetivos son, entre otros, mejorar la capacidad exportadora y competitiva de esos sectores. En ese mismo contexto se enmarca el proyecto de "incubadora de empresas", que persigue establecer un espacio inicial para proyectos de alumnos que pudieran generar nuevas empresas. Este plan tienen dos áreas de desarrollo: "Genera UC", establecido en la Facultad de Ingeniería, y "Ventana UC", con el área de la educación.

10 El cambio de perspectiva desde temas genéricos a situaciones de interés reconocible ha duplicado el número de ejemplares de su tirada (de 2.400 a 5.200), y ha hecho crecer las suscripciones pagadas de 1.600 a más de 4.000. La universidad reparte gratuitamente 1.200 ejemplares para sus profesores y para líderes de opinión, que se financian íntegramente con los ingresos generados por la propia revista.

11 Se han realizado cursos de capacitación para pequeños y medianos empresarios en convenio con el Banco de Chile y el Banco Estado, cursos para la Fuerza Aérea, CODELCO y CONAF, y programas de capacitación para profesores de párvulos y de la asignatura inglés, en conjunto con el Ministerio de Educación. 
Recientemente, a través de la Fundación Copec-UC, se han premiado con fondos para investigación algunas iniciativas (no necesariamente radicadas en la PUC, sino en todas las universidades del país) que pueden resultar señeras en la perspectiva de desarrollar las riquezas propias. Entre ellas está la producción y comercialización de biltong de guanaco, la valorización económica del luche y los aceites esenciales de alta calidad, entre otros.

El trabajo con las empresas tiene también otras ventajas desde el aspecto universitario: los productos naturales (en su uso industrial o farmacéutico), los alimentos, las experiencias agrícolas en condiciones especiales, la minería, la resistencia de materiales y los movimientos telúricos, además de los estudios climáticos en zonas geográficas diversas, constituyen algunos ejemplos de alternativas de conocimiento en las cuales Chile presenta ventajas comparativas frente a otros países. Los logros de investigación y desarrollo en estos campos aumentan también la autovaloración al interior del país, y contribuyen a la expectativa de un diálogo cultural en condiciones más igualitarias con los países más desarrollados. Además, es el tipo de conocimiento que alumnos y profesores extranjeros pudieran venir a buscar aquí, lo cual redunda en una corriente de intercambio de vastas proporciones.

Dentro de la relación con los diversos actores sociales, la universidad ha reforzado también el contacto con las municipalidades. Entre estas iniciativas cabe señalar el acuerdo con la I. Municipalidad de Pirque para el programa PENTA UC destinado a los niños con talento, con la I. Municipalidad de Macul para la administración de la Escuela Julio Barrenechea, con la I. Municipalidad de Providencia para actividades culturales en el Campus Oriente, o proyectos de itinerancia con conferencias, teatro y música para diversas municipalidades a través del país. También puede enmarcarse en este contexto la Red de Salud dependiente de la Facultad de Medicina y la iniciación reciente de consultorios periféricos de atención primaria en salud.

3. Las variadas formas de acercamiento temático, propiciando la mirada pluridisciplinaria de problemas complejos

En este aspecto, la Pontificia Universidad Católica ha creado instancias destinadas a conocer procesos de cambio en áreas significativas del desarrollo nacional -tales como la vivienda social, el diseño de software, textos y otros materiales para escolares, y la conservación del medio 
ambiente, entre otras iniciativas-, y propiciar respuestas válidas para ellos. Cabe destacar, en este aspecto, el concurso internacional para desarrollar un nuevo proyecto de vivienda social, en el que participaron arquitectos de diez países del mundo, y el Centro de Informática Educativa, por ejemplo, donde convergen las áreas de Ingeniería y Educación.

Debido a la investigación y al trabajo de extensión y ayuda a las personas que ellas realizan, tiene particular relevancia la presencia de iniciativas académicas como el "Programa de Estudios sobre la Familia", el "Centro de Bioética", el "Programa de Doctrina Social" y el "Centro de Estudios Médicos Humanísticos", por mencionar algunos.

\section{Transferencia de conocimientos a grupos extensos}

El trabajo en este ámbito ha consistido en potenciar una línea de educación continua que aporte conocimiento de alto nivel universitario a un público alternativo, especialmente constituido por profesionales, y se avanza hacia programas sustentados en tecnologías a distancia.

En el futuro se espera consolidar un modelo que represente efectivamente una educación a lo largo de la vida y que sea eficaz en una sociedad en la cual, por la rapidez con que varía el conocimiento, ya no puede ofrecerse sólo una formación única al inicio de la vida adulta.

Las facultades han sido muy conscientes de la urgencia de este camino. En estos últimos cuatro años los programas de diplomados han crecido en un $100 \%$ y se han orientado, de modo claro, hacia los intereses de quienes se encuentran plenamente incorporados a la vida del trabajo (ver Cuadro 2). Una experiencia exitosa en el campo del trabajo a distancia lo constituyen los diplomados de "Clase Ejecutiva", dictados en combinación con el diario El Mercurio.

Cuadro 2

\begin{tabular}{|l|r|r|r|r|}
\hline Diplomados & 2000 & 2001 & 2002 & 2003 \\
\hline Programas nuevos & 12 & 19 & 23 & 29 \\
\hline Programas activos & & 40 & 61 & 80 \\
\hline Alumnos inscritos & & 1.060 & 1.891 & 2.819 \\
\hline Alumnos / Programa & & 27 & 31 & 35 \\
\hline
\end{tabular}


Dentro de esta línea de trabajo se encuentra también el Preuniversitario $\mathrm{UC}^{12} \mathrm{y}$, de manera destacada, el Programa del Adulto Mayor, que establece nuevas modalidades de acción para personas de la tercera edad. El proyecto más reciente está destinado a crear capacidades empresariales en personas mayores para contribuir al mejoramiento de su calidad de vida (ver Cuadro 3).

Cuadro 3

\begin{tabular}{|c|c|c|}
\hline Programa de transferencia 2003 & $\mathrm{N}^{\circ}$ de cursos & $\mathrm{N}^{\circ}$ de alumnos \\
\hline Teleduc: & 41 & 12.626 \\
\hline Preuniversitario & 171 & 4.397 \\
\hline Adulto Mayor & 56 & 1.681 \\
\hline
\end{tabular}

Cabe recordar, también, que la Pontificia Universidad Católica cuenta con una editorial que publica textos especializados y que tiene, adicionalmente, una línea destinada a la educación secundaria y otra a público general. Por último, parece necesario hacer referencia a la constante organización de espacios internacionales de encuentro, discusión y síntesis de experiencia, a través de seminarios y congresos $^{13}$, y -en un plano diferente- la publicación de una "Guía de Expertos"14.

\section{Para finalizar}

Hay un último elemento de análisis de la significación de la extensión desarrollada en la perspectiva señalada anteriormente, y que sólo puede dejarse en un plano de enunciado: la situación geográfica de aislamiento que tiene Chile contrasta con su apertura cultural y económica. El país está formado, culturalmente, por diversas corrientes migratorias que han traído consigo algunas de sus raíces y que

12 El Preuniversitario tiene cinco sedes en Santiago y una pequeña sede en Rancagua.

13 Entre los simposios y congresos de carácter internacional cabe destacar, por último, el Simposio Internacional de Políticas Culturales, el organizado con ocasión del Año del Patrimonio Cultural de la UNESCO, Diálogo Norte-Sur, Desafíos de la Educación en América Latina, el Congreso Internacional sobre Derecho Constitucional, El Congreso Mundial de Educación, y otros; todos con más de quinientos participantes.

14 Esta guía, en su segunda edición, entrega una lista de cerca de mil profesores, ordenada por nombre y materia de especialidad, para que pueda ser consultada de modo eficiente. La primera edición, de 585 nombres, se agotó en menos de un año. 
constituyen una amalgama de bases menos difundidas que culturas establecidas sobre civilizaciones indígenas arraigadas, como es el caso de México, Perú o Guatemala, por ejemplo. Además, una proporción de la población se vio repentinamente expuesta a vivencias en culturas de otras latitudes, como resultado del exilio y las migraciones en los gobiernos de la Unidad Popular y militar. Ese contexto ha generado desigualdades de experiencias y raíces culturales donde la universidad puede jugar un importante rol de nexo, reconocimiento y vínculo permanente, lo que se suma a los objetivos antes descritos. 